\title{
Método computacional baseado em workflow para contabilização da frequência de repetição de $\boldsymbol{k}$-mers
}

\author{
Fabrício Vilasbôas ${ }^{1}$, Carla Osthoff $^{1}$, Kary Ocaña ${ }^{1}$, Oswaldo Trelles ${ }^{2}$, Ana Tereza Vasconcelos ${ }^{1}$ \\ ${ }^{1}$ Laboratório Nacional de Computação Científica (LNCC)
}

${ }^{2}$ Universidad de Málaga

\begin{abstract}
Resumo. Este trabalho apresenta uma análise do desempenho do SCFRK, um algoritmo determinístico para uma aplicação de bioinformática computacionalmente intensiva, o k-mer, para uma arquitetura GPGPU em um ambiente de workflow científico. Nossos experimentos demonstram que o SCFRK é uma alternativa eficiente e de baixo custo para a contabilização de k-mers para análises em metagenoma.
\end{abstract}

\section{Introdução}

$\mathrm{O}$ advento de novas tecnologias de sequenciamento na área de bioinformática está fazendo com que o gargalo migre da aquisição de dados para o processamento e interpretação dos mesmos. Por outro lado, novas tecnologias de processamento de alto desempenho de baixo custo tais como processadores gráficos, GPU's [Kirk and Wen-mei 2012], podem ser utilizadas para aumentar a taxa de processamento dos dados.

Em recentes trabalhos, [Vilasboas et al. 2015] e [Vilasboas et al. 2016], foi apresentado o algoritmo CFRK, desenvolvido para a contabilização da frequência de repetição de $k$-mers em ambiente $G P U$ que apresenta bom desempenho para aplicações com valores de $\mathrm{k}$ menor ou igual a 5 , tal como metagenoma. Neste trabalho apresentamos a avaliação de desempenho do CFRK em um ambiente de workflow cientifico, o Swift, um SWfMS (Scientific Workflow Management Systems) contendo GPU's. Na Seção 2 apresentamos os trabalhos relacionados. Na Seção 3 apresentamos a descrição do SCFRK. $\mathrm{Na}$ Seção 4 apresentamos os experimentos e os resultados. Na Seção 5 apresentamos as conclusões e os trabalhos futuros.

\section{Trabalhos relacionados}

Nesta seção relacionaremos o algoritmo SCFRK com o Jellyfish, um algoritmo desenvolvido para processamento de $k$-mers e que é considerado o estado da arte nesta tarefa.

O Jellyfish [Marçais and Kingsford 2011] é um algoritmo para contabilização da frequência de repetição de $k$-mers específico para genomas desenvolvido para processamento em memória compartilhada. Ele utiliza várias estruturas lock-free, que são estruturas que permitem operações atômicas sem o bloqueio da memória e, por isso, não degradam o desempenho em ambientes multiprocessáveis. A diferença mais importante entre os algoritmos Jellyfish e SCFRK é que o SCFRK foi desenvolvido para o processamento de metagenoma, enquanto o Jellyfish foi feito para o processamento de genoma. $\mathrm{O}$ 
Jellyfish considera todos os reads como provenientes de um mesmo organismo, o SCFRK considera cada read como sendo de organismos distintos. Isso permite que o SCFRK seja de propósito mais geral.

\section{SCFRK}

SCFRK é o acrônimo de $\boldsymbol{S}$ wift - Contabilizador da Frequência de Repetição de K-mers, pois foi desenvolvido utilizando os recursos do Swift.

A execução do SCFRK é dividida em duas fases bem definidas: o préprocessamento e o processamento. Na fase de pré-processamento é realizada a divisão do arquivo que contém os reads. É utilizado uma aplicação em linguagem $\mathrm{C}$ que realiza o particionamento do arquivo em $\mathrm{N}$ partes iguais, onde $\mathrm{N}$ é um valor definido pelo usuário. Na fase de processamento, o SCFRK é responsável pelas chamadas ao CFRK. É fornecido para cada processo a localização do arquivo de entrada e do arquivo de saída. Ao final do processamento, os arquivos de saída são escritos em disco.

A principal motivação para o desenvolvimento do SCFRK se deve à limitação da execução do CFRK para arquivos de entrada com tamanho igual ou superior ao tamanho da memória principal da estação de trabalho em uso. Ao ler um arquivo de entrada de tamanho igual ou superior ao tamanho da memória principal, a estação de trabalho poderá entrar em processo de paginação ou poderá entrar em colapso se não houver espaço em disco para a paginação. Esta estratégia de paralelismo permite a divisão do arquivo de entrada em arquivos que sejam do tamanho adequado para o processamento na estação de trabalho.

\section{Experimentos}

Nesta seção serão apresentados os experimentos e resultados da execução do SCFRK, bem como algumas comparações com o algoritmo Jellyfish.

\subsection{Ambiente de teste}

Para os testes foram utilizados quatro nós da cluster Altix-Xe do CENAPAD/LNCC. Cada nó possui dois processadores Intel(R) Xeon(R) CPU E5-2660 0 @ $2.20 \mathrm{GHz}$ com 8 núcleos cada processador e uma GPU Nvidia Tesla K20m com 5 GB de memória $R A M$ e 2496 núcleos. O sistema operacional utilizado foi o CetOS, kernel versão 2.6.32-573.7.1.el6.x86_64.

$\mathrm{O}$ arquivo de entrada utilizado foi uma amostra de metagenoma de um ambiente real obtida no banco de dados SRA do NCBI (National Center for Biotechnology Information), cujo ID nesta base de dados é SRX2021688. Este arquivo contém 8.7GB de dados e 27017895 reads.

\subsection{Resultados}

Os experimentos consistiram em executar os algoritmos SCFRK e Jellyfish com o mesmo conjunto de dados. Os valores de $k$ foram selecionados baseados em trabalhos anteriores [Vilasboas et al. 2015] que demonstraram que o CFRK apresenta ganho em relação ao Jellyfish para valores de $k<5$. O tempo total de execução foi obtido através do comando time do linux, sendo considerado apenas o tempo real. Para este trabalho foi 
considerado apenas o tempo da fase de processamento do SCFRK, pois o foco da análise é a execução do módulo desenvolvido utilizando o Swift e o Jellyfish não possui a etapa de divisão do arquivo. Cada processo lançado para a execução do workflow receberá o nome de worker.

Para o algoritmo SCFRK foram utilizados: dois nós com um worker por nó; três nós com um worker por nó; quatro nós com um worker por nó; e quatro nós com dois workers por nó. Para o Jellyfish foi utilizado um nó com oito threads.

A Figura 1 apresenta o tempo total de execução dos algoritmos Jellyfish e SCFRK. As barras em azul apresentam o tempo total de execução do Jellyfish para $k=2,3,4,5$. As barras em laranja apresentem o tempo de execução do SCFRK com 2 workers para $k=2,3,4$. As barras em amarelo apresentem o tempo de execução do SCFRK com 3 workers para $k=2,3,4,5$. As barras em verde apresentem o tempo de execução do SCFRK com 4 workers para $k=2,3,4,5$. As barras em vinho apresentem o tempo de execução do SCFRK com 8 workers para $k=2,3,4,5$.

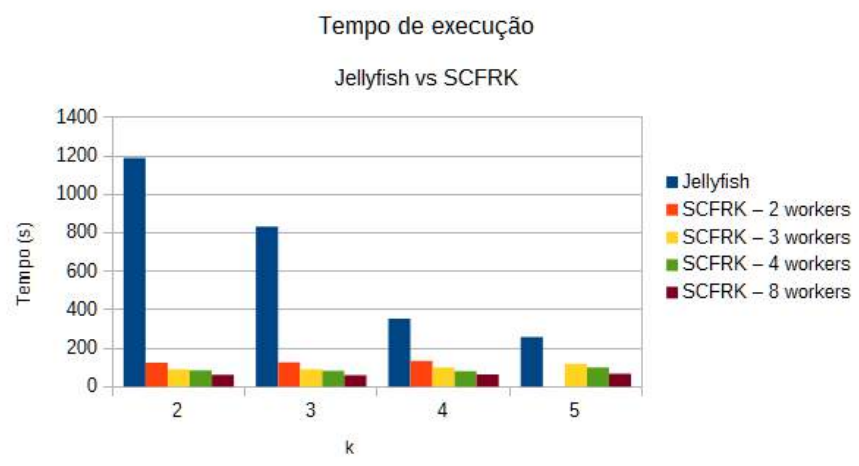

Figure 1. Tempo de execução dos algoritmos SCFRK e Jellyfish

A Figura 2 apresentam o ganho obtido pelo SCFRK em relação ao Jellyfish. A linha em azul apresenta o ganho do SCFRK utilizando 2 workers. A linha em laranja apresenta o ganho do SCFRK utilizando 3 workers. A linha em amarelo apresenta o ganho do SCFRK utilizando 4 workers. A linha em verde apresenta o ganho do SCFRK utilizando 8 workers.

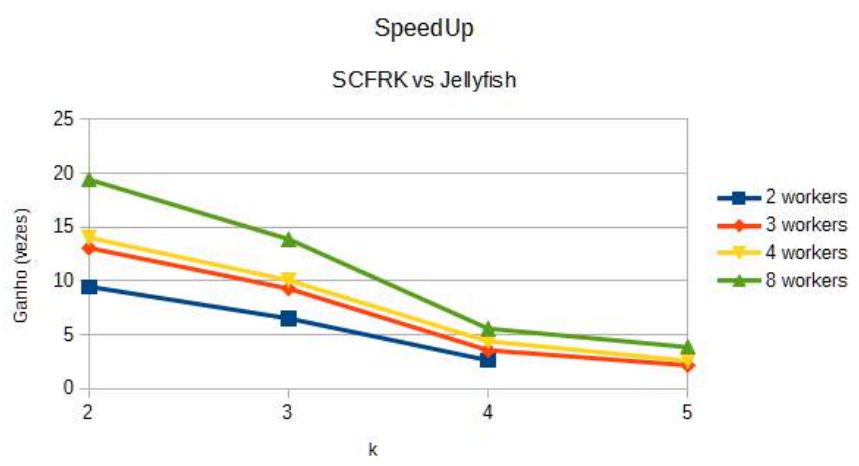

Figure 2. Ganho obtido pelo SCFRK em relação ao Jellyfish 


\subsection{Discussão do resultado}

Ao observar a Figura 1 vemos que o SCFRK apresenta um menor tempo de execução em relação ao Jellyfish para todos os casos. Observamos que o tempo de execução de SCFRK se mantém praticamente constante com o aumento do valor de $k$. Isso acontece por conta da característica do algoritmo CFRK que processa todas as combinações de forma independente e, dessa forma, mais threads são ativadas e aumenta a ocupação da $G P U$. Outra observação é em relação ao tempo de execução do Jellyfish. Vemos que a medida que o valor de $k$ aumenta o tempo de execução decai. Isto acontece porque o Jellyfish foi desenvolvido para o processamento de um genoma, ou seja, ele considera todos os reads como pertencentes a um mesmo organismo. O Jellyfish aloca um único vetor global para contabilizar a frequência de repetição dos k-mers de todos os reads. Portanto, a medida que o valor de $k$ aumenta ele manipula menos a memória, dado que o número de possíveis combinações decai com o aumento do valor de $k$.

A Figura 2 mostra que para $k=2$ o menor ganho do SCFRK foi de 9.4 vezes com 2 workers e o maior ganho foi de 19.4 vezes com 8 workers. Para $k=3$ observamos que o menor ganho foi de 6.5 vezes com 2 workers e o maior ganho foi de 13.9 com 8 workers. Para $k=4$ temos que o menor ganho foi de 2.6 vezes com 2 workers e o maior ganho foi de 5.5 vezes com 8 workers. Para $k=5$ o menor ganho foi de 2.2 vezes com 3 workers e o maior ganho foi de 3.8 vezes com 8 workers.

\section{Conclusão e trabalhos futuros}

Neste trabalho foi apresentado o SCFRK, uma variação do algoritmo CFRK utilizando o gerenciador de workflow cientifico Swift. Mostramos que o SCFRK apresenta bom desempenho com ganho máximo de 19.4 vezes para $k=2$ com 8 workers e ganho mínimo de 2.2 vezes com 3 workers para $k=5$ em relação ao Jellyfish e que é uma opção viável para a contabilização de $k$-mers menores que 5 em aplicações tais como de metagenoma em ambientes de workflow cientifico com GPU's. Como trabalhos futuros pretendemos avaliar o desempenho com grandes bases de dados em ambientes computacionais que permitam implementar um grande número de workers, tal como o supercomputador SDumont.

\section{References}

Foster, I. (1994). Task parallelism and high-performance languages. IEEE Parallel \& Distributed Technology: Systems \& Technology, 2(3):27-36.

Kirk, D. B. and Wen-mei, W. H. (2012). Programming massively parallel processors: a hands-on approach. Newnes.

Marçais, G. and Kingsford, C. (2011). A fast, lock-free approach for efficient parallel counting ofoccurrences of k-mers. Bioinformatics (Oxford, England), 27(6):764-70.

Vilasboas, F., Osthoff, C., Trelles, O., and Vasconcelos, A. T. (2015). Desenvolvimento de um algoritmo paralelo para contabilização da repetição de k-mers. $3^{a}$ Conferência Ibero Americana de Computação Aplicada 2015.

Vilasboas, F., Osthoff, C., Trelles, O., and Vasconcelos, A. T. (2016). Otimização de um algoritmo paralelo para contabilização da repetição de k-mers. II Escola Regional de Computação de Alto Desempenho do Rio de Janeiro. 